\title{
Lack of substrate inhibition in a monomeric form of human cytosolic SULT2A1
}

\author{
Ian T. Cook ${ }^{1}$, Thomas S. Leyh², Susan A. Kadlubar ${ }^{3}$, and Charles N. Falany ${ }^{1, *}$ \\ ${ }^{1}$ Department of Pharmacology and Toxicology, University of Alabama at Birmingham, \\ Birmingham, AL, USA \\ ${ }^{2}$ Department of Microbiology and Immunology, Albert Einstein College of Medicine, Bronx, NY, \\ USA \\ ${ }^{3}$ Department of Environmental and Occupational Health Sciences, University of Arkansas for \\ Medical Sciences, Little Rock, AR, USA
}

\begin{abstract}
Mammalian cytosolic sulfotransferases (SULTs) frequently show substrate inhibition during the sulfation of increasing concentrations of substrates. SULT2A1, a major human liver isoform responsible for the conjugation of hydroxysteroids, bile acids and aliphatic hydroxyl groups in drugs and xenobiotics, is a homodimer and displays substrate inhibition during the conjugation of dehydroepiandrosterone (DHEA). Maltose binding protein (MBP)-SULT2A1 fusion protein, produced as an intermediate step in the purification of the SULT2A1 homodimer, elutes during size exclusion chromatography as a monomer. The initial-rate parameters $\left(K_{\mathrm{m}}\right.$ and $\left.V_{\max }\right)$ of the monomer (MBP-SULT2A1) and native SULT2A1 dimer for DHEA sulfation are extremely similar; however, the monomer is not inhibited by DHEA. Intrinsic fluorescence studies show that two DHEA molecules bind each SULT2A1 subunit, one in the catalytic site and one in an apparent allosteric site. Lack of dimerization in the MBP-SULT2A1 fusion protein decreased the $K_{\mathrm{d}}$ for binding of DHEA at the allosteric site. These results suggest that formation of the homodimer is associated with structural rearrangements leading to increased DHEA binding at an allosteric site that is associated with substrate inhibition.
\end{abstract}

\section{Keywords}

dehydroepiandrosterone; dimerization; maltose binding protein; substrate inhibition; sulfation; sulfotransferase; SULT2A1

\section{Introduction}

Sulfotransferase (SULT) 2A1 is a major SULT isoform expressed in human liver and is responsible for the sulfation of a wide range of hydroxysteroids, sterols and bile acids (1-3). This human SULT isoform has also been termed dehydroepiandrosterone (DHEA)-SULT owing to its involvement in the synthesis of high levels of DHEA-sulfate in the reticular layer of the adrenal cortex $(4,5)$. Consistent with its role as a xenobiotic conjugating enzyme in the liver and GI tract, SULT2A1 has a broad reactivity with aliphatic hydroxyl

\footnotetext{
(C) 2010 by Walter de Gruyter

*Corresponding author: Charles N. Falany, Department of Pharmacology and Toxicology, 1670 University Blvd., Volker Hall G133M, University of Alabama at Birmingham, Birmingham, AL 35294, USA, Phone: +1-205-934-9848, Fax: +1-205-934-9888,

cfalany@uab.edu.
} 
groups in many drugs and xenobiotics $(3,5,6)$. SULT2A1 expression in human liver is also regulated by several of the xenobiotic-sensing nuclear receptors $(7,8)$.

Most cytosolic SULTs show substrate inhibition during the conjugation of increasing concentrations of substrates, particularly compounds that represent higher affinity substrates $(2,3,5)$. A single SULT isoform can display substrate inhibition with one compound but not demonstrate substrate inhibition with a different substrate (9). SULT2A1 displays substrate inhibition during the conjugation of increasing concentrations of DHEA with maximal activity observed at a DHEA concentration of approximately $3 \mu \mathrm{M}$ (2). In contrast, SULT2A1 also readily conjugates raloxifene yet substrate inhibition is not observed with concentrations up to $20 \mu \mathrm{M}$ (9). As observed with other SULTs during substrate inhibition, as the concentration of the sulfonate acceptor substrate increases (e.g., DHEA), the declining activity plateaus at a lower but constant rate indicating partial substrate inhibition (10). Substrate inhibition in the human SULTs has been attributed to several mechanisms including formation of non-productive ternary enzyme complexes, allosteric binding and multiple substrates binding in the active site (10-12).

Cook et al. (9) have recently reported that 3'-phosphoa-denosine 5'-phosphosulfate (PAPS) binding to SULT2A1 results in a structural rearrangement in the peptide (loop3) forming the outer surface of the active site. PAPS binding decreases the volume of the substrate-binding pocket and significantly alters selectivity for binding of substrates to the enzyme. DHEA can readily bind to SULT2A1 in the presence or absence of PAPS, whereas raloxifene can bind only in a catalytic conformation in the absence of PAPS binding. The motile loop3 is contiguous with the KTVE domain, a highly conserved motif proposed as the dimerization domain in most SULT isoforms $(13,14)$ suggesting an interaction between dimerization and the regulation of sulfation activity.

Our laboratory has used the pMAL expression system to generate a MBP-SULT2A1 fusion protein that can subsequently be cleaved to produce pure, native SULT2A1 $(9,15)$. One advantage of the pMAL system is that the MBP-SULT2A1 fusion protein is enzymatically active allowing for easier detection and quantitation of enzyme activity during the purification procedure. However, the addition of the MBP moiety to the amino-terminus of SULT2A1 disrupts homodimer formation, as demonstrated by the elution of MBPSULT2A1 during gel exclusion chromatography. Characterization of the sulfation of increasing DHEA concentrations by the MBP-SULT2A1 monomer does not result in substrate inhibition. The lack of observable substrate inhibition with the MBP-SULT2A1 monomer implicates dimerization of SULT2A1 as having a role in regulating the reaction mechanism. Therefore, the kinetic properties of the MBP-SULT2A1 fusion protein were compared with the native SULT2A1 enzyme to investigate the structure/function relationships associated with substrate inhibition in SULT2A1.

\section{Materials and methods}

\section{Materials}

DHEA was purchased from Sigma Chemical Co. (St Louis, MO, USA). PAPS was obtained from Dr Sanford Singer (University of Dayton, Dayton, OH). [ ${ }^{35}$ S]PAPS and $\left[1,2,6,7-{ }^{3} \mathrm{H}(\mathrm{N})\right]$ DHEA were purchased from Perkin-Elmer (Waltham, MA, USA). LK6DF 60 A silica gel thin-layer chromatography (TLC) plates with a layer thickness of $250 \mu \mathrm{m}$ were obtained from Whatman Inc. (Cliffton, NJ, USA). The Molecular Operating Environment platform (MOE) was accessed with a license provided by Chemical Computing Group (Montreal, Quebec, Canada). All other chemicals used were reagent grade purchased from Fisher Scientific (Norcross, GA, USA). 


\section{Expression and purification of SULT2A1}

The SULT2A1 cDNA was cloned into the XmnI and HindIII sites of the pMAL-c2 vector to generate a transcript that translates into a MBP-SULT2A1 fusion protein with a Factor Xa protease cleavage site immediately before the initial Met in SULT2A1 (15). pMALSULT2A1 was expressed in E. coli XL1-Blue cells under ampicillin selection, and lysate prepared from the cells by sonication. The cytosolic fraction containing SULT2A1 activity was recovered following centrifugation at $100,000 \times g$ for $1 \mathrm{~h}$ at $4^{\circ} \mathrm{C}$. The MBP-SULT2A1 fusion protein was purified by amylose affinity chromatography using $10 \mathrm{mM}$ maltose to elute the MBP-SULT2A1 protein from the amylose affinity column. Because the MBPSULT2A1 fusion protein was capable of sulfating DHEA, enzyme activity was used to monitor protein purification. To isolate SULT2A1 from MBP-SULT2A1, the fusion protein was digested with Factor Xa protease (New England Biolabs, Ipswich, MA, USA), and the Factor Xa protease was removed using DEAE-Sepharose CL-6B chromatography. The cleaved MBP-SULT2A1 preparation was loaded on the DEAE-Sepharose column and the column was washed with $5 \mathrm{mM}$ phosphate $\mathrm{pH} 7.4$ buffer followed by two column volumes of buffer containing $100 \mathrm{mM} \mathrm{NaCl}$. SULT2A1 activity was then eluted with a $100-225 \mathrm{mM}$ $\mathrm{NaCl}$ gradient in the same buffer (2). Active SULT2A1 elutes at approximately $140 \mathrm{mM}$ $\mathrm{NaCl}$. To remove residual amounts of the uncut fusion protein and free MBP, the active SULT2A1 fraction was passed through a second amylose column. The purified SULT2A1 was then analyzed by SDS-PAGE and assayed for activity.

\section{DHEA sulfation assay}

DHEA sulfation assays were performed as described previously using $\left[{ }^{3} \mathrm{H}\right]$ DHEA and nonradiolabeled PAPS (2). Reactions were terminated with the addition of $\mathrm{CHCl}_{3}$ and alkalinized with the addition of $0.25 \mathrm{M}$ Tris- $\mathrm{HCl}, \mathrm{pH} 8.8$. The $\left[{ }^{3} \mathrm{H}\right] \mathrm{DHEA}$-sulfate formed remained in the aqueous phase and was quantified by scintillation counting. To detect substrate inhibition, the effect of increasing DHEA concentrations on DHEA sulfation with SULT2A1 was determined in the presence of high PAPS concentrations. The effects of DHEA concentrations on substrate activation by increasing PAPS concentrations were also analyzed. For substrate activation studies, DHEA sulfation at concentrations of 2 and $20 \mu \mathrm{M}$ DHEA was assayed with increasing PAPS concentrations.

The kinetic parameters of the DHEA sulfation reaction were analyzed using four concentrations each of PAPS and DHEA in a $4 \times 4$ format with each combination of DHEA and PAPS run in triplicate. $K_{\mathrm{m}}$ and $V_{\max }$ values were estimated from the replots of the slopes and intercepts (16).

\section{Stoichiometry and dissociation constant $\left(K_{\mathrm{d}}\right)$ estimations}

The stoichiometry and $K_{\mathrm{d}}$ values for DHEA and PAP/PAPS binding to SULT2A1 were estimated using changes in intrinsic fluorescence of the pure protein. These studies were performed with pure SULT2A1 using a Perkin-Elmer LS-5 fluorescence spectrophotometer with excitation at $275 \mathrm{~nm}$ and detection at $340 \mathrm{~nm}$. For stoichiometry studies, $10 \mu \mathrm{M}$ SULT2A1 subunits in $10 \mathrm{mM}$ phosphate buffer, $\mathrm{pH} 7.4$ with $150 \mathrm{mM} \mathrm{NaCl}$ and $1 \mathrm{mM}$ DTT in a volume of $3 \mathrm{~mL}$ were used. Increasing amounts of DHEA or PAP/PAPS were added in $2 \mu \mathrm{L}$ aliquots and allowed to equilibrate for $3 \mathrm{~min}$ before fluorescence was measured. The change in fluorescence was plotted against the ratio of substrate to SULT2A1 subunit concentration. The point at which the fluorescence change ceases represents saturation of the binding site(s). To measure substrate binding to the closed conformation of SULT2A1, the enzyme was preincubated with $20 \mu \mathrm{M}$ PAP before to titration with DHEA. The effect of DHEA on the binding of PAP to SULT2A1 was also analyzed after preincubation of the enzyme with an excess of substrate (e.g., $20 \mu \mathrm{M}$ DHEA). PAP rather than PAPS was used in the studies with DHEA binding because PAPS would allow for DHEA sulfation. 
For $K_{\mathrm{d}}$ determinations, the SULT2A1 subunit concentration was $100 \mathrm{nM}$ to stay below the $K_{\mathrm{m}}$ values for either substrate. Titrations with increasing substrate concentrations were performed in a manner similar to the stoichiometry studies. The $K_{\mathrm{d}}$ values were calculated from the double reciprocal plots of fluorescence change vs. substrate concentration where the $K_{\mathrm{d}}$ was determined from the $X$-intercept as $-1 / K_{\mathrm{d}}$. The $K_{\mathrm{d}}$ values of both substrates and PAP were also determined using enzyme preincubated with a saturating excess of the other substrate to observe the effects of substrate-induced conformational changes.

\section{Results}

Generation of MBP-SULT2A1 fusion protein is used as a step in the purification of the active native form of SULT2A1 (15). The ability of the fusion protein to catalyze DHEA sulfation activity is also useful in following purification of SULT2A1 activity. To generate native SULT2A1, the MBP-SULT2A1 fusion protein was cleaved with Factor Xa and SULT2A1 purified in several steps (15). The pure native SULT2A1 derived from cleavage of the MBP-SULT2A1 fusion protein elutes during Sephadex G-100 chromatography with a mass consistent with formation of a homodimer (Figure 1A). By contrast, when the MBPSULT2A1 fusion protein was run on the same gel exclusion column, both protein and DHEA sulfation activity eluted with a molecular mass consistent with that of an MBPSULT2A1 monomer (Figure 1B). Although the MBP-2A1 fusion protein (MW= $78 \mathrm{kDa})$ eluted slightly before dimeric SULT2A1 $(\mathrm{MW}=70 \mathrm{kDa})$ there was no significant difference in their elution volumes (Figure 1). In both procedures immunoreactive SULT2A1 protein was detected only in fractions with detectable DHEA sulfation activity.

Most of the cytosolic SULTs exist as homodimers and are proposed to use a highly conserved 'KTVE' binding motif as the dimerization interface (13, 17). Although several dimer structures for SULT2A1 have been resolved, these structures utilize different dimer interfaces and apparently represent crystallization artifacts $(12,18,19)$. To better evaluate the possible implications of dimerization on the activity of SULT2A1, MOE was utilized to generate a model of the expected SULT2A1 homodimer. Figure 2 shows the model of SULT2A1 utilizing the KTVE region as the dimerization interface. The KTVE domain is contiguous with the loop3 peptide that was recently reported to rearrange to limit access of selected substrates to the active site when PAPS is bound (9). Figure 3 shows a graph of the root mean squared fluxuation (RMSF) calculation for SULT2A1 with the highest RMSF for the loop3 domain. Loop3 forms the outer surface of the active site and is capable of moving more than $1.0 \mathrm{~nm}$ when PAPS binds thereby decreasing the volume of the substrate binding pocket approximately $40 \%$. PAPS binding also results in a smaller $0.2 \mathrm{~nm}$ rearrangement in the KTVE domain although the rearrangement does not affect dimerization. Elution of the MBP-SULT2A1 fusion protein as a monomer suggests that the presence of the MBP moiety at the amino-end of the protein interferes with the dimerization process without significantly obstructing access to either the PAPS or DHEA binding site in the catalytic site.

Because the MBP-SULT2A1 fusion protein was an apparent monomer, the kinetic properties of its DHEA sulfation activity were examined to more clearly define activity levels during the purification procedure. Substrate inhibition with increasing concentrations of DHEA has been reported with pure human liver dimeric SULT2A1 (2). The pure SULT2A1 generated by cleavage of the MBP-SULT2A1 fusion protein with Factor Xa also demonstrated substrate inhibition (Figure 4). By contrast, the MBP-SULT2A1 fusion protein used to generate the native SULT2A1 does not exhibit substrate inhibition during the sulfation of increasing DHEA concentrations. To further investigate whether monomeric forms of SULT might not show substrate inhibition, the naturally occurring monomeric mouse SULT1E1 was assayed for substrate inhibition during $\beta$-estradiol (E2) sulfation (17). Figure 5 shows that mouse hepatic SULT1E1 does not demonstrate substrate inhibition at 
E2 concentrations at greater than 15 -fold its $K_{\mathrm{m}}$ of $6 \mathrm{nM}(20,22)$. We have previously reported that human SULT1E1, which is a dimer, shows potent substrate inhibition during E2 sulfation with maximal activity at $15-20 \mathrm{nM}$ and a $K_{\mathrm{m}}$ of $4 \mathrm{nM}(10,21)$.

Although the MBP-SULT2A1 fusion protein does not demonstrate substrate inhibition during DHEA sulfation, the kinetic and binding properties of the active site do not appear to be affected. The $K_{\mathrm{m}}$ and $V_{\max }$ values for PAPS and DHEA calculated for both the MBPSULT2A1 fusion protein and the pure native SULT2A1 were similar. The $K_{\mathrm{m}}$ for DHEA was estimated as $1.6 \pm 0.1 \mu \mathrm{M}$ using the MBP-SULT2A1 fusion protein and $1.4 \pm 0.1 \mu \mathrm{M}$ with pure dimeric SULT2A1 generated by cleavage of the MBP-SULT2A1. The $V_{\max }$ rates calculated for both the MBP-2A1 fusion protein and the pure SULT2A1 were 3.2 \pm 0.2 and $3.5 \pm 0.3 \mathrm{nmol} / \mathrm{min}$ per $\mathrm{mg}$, respectively. The similar $K_{\mathrm{m}}$ and $V_{\max }$ values indicate that the kinetics of substrate binding to the active site nor the reaction mechanism are significantly affected by the lack of subunit interaction.

To further examine the binding properties of the active sites of both the MBP-SULT2A1 fusion protein and pure SULT2A1, the stoichiometry of subunit binding and dissociation constants $\left(K_{\mathrm{d}}\right)$ for PAPS/PAP and DHEA binding were determined. Figure 6A,B shows that two DHEA molecules can bind in each subunit of both pure SULT2A1 and the MBPSULT2A1 fusion protein in the absence of PAP. However, if both SULT2A1 and MBPSULT2A1 were incubated with PAP before adding DHEA then binding of only one DHEA molecule was detected (Figure 6C,D). Both the MBP-SULT2A1 fusion protein and pure SULT2A1 bound only one molecule of PAP per monomer in the presence or absence of DHEA (data not shown).

The $K_{\mathrm{d}}$ values for the binding of substrates to both the MBP-SULT2A1 fusion protein and pure SULT2A1 were determined using changes in intrinsic fluorescence elicited by the addition of increasing amounts of either PAPS or DHEA. The $K_{\mathrm{d}}$ for DHEA was also estimated in the presence of the enzymes preincubated with excess PAP. Table 1 shows the estimated $K_{\mathrm{d}}$ values. DHEA binding to pure SULT2A1 was resolved into two curves with $K_{\mathrm{d}}$ values of $290 \pm 40 \mathrm{nM}$ and $3100 \pm 500 \mathrm{nM}$. By contrast, only one $K_{\mathrm{d}}$ of $280 \pm 20 \mathrm{nM}$ was estimated for DHEA binding to the monomeric MBP-SULT2A1 fusion protein, although the stoichiometry studies indicated that two DHEA molecules were binding per subunit. Following preincubation with excess PAP, only one $K_{\mathrm{d}}$ was also determined for PAPS binding to either pure SULT2A1 $(350 \pm 30 \mathrm{nM})$ or the MBP-SULT2A1 fusion protein $(390 \pm 60 \mathrm{nM})$. The $K_{\mathrm{d}}$ values for both PAPS and PAP binding were estimated with both SULT2A1 and MBP-SULT2A1. For PAPS binding to both enzymes only a single $K_{\mathrm{d}}$ was observed and the estimated $K_{\mathrm{d}}$ values were very similar (Table 1). PAP binding to both SULT2A1 and MBP-SULT2A1 also showed a single $K_{\mathrm{d}}$ value that was slightly higher than that the $K_{\mathrm{d}}$ for PAPS binding.

Because substrate inhibition is prominent in the sulfation of DHEA by SULT2A1, most kinetic studies of SULT2A1 are conducted at relatively low DHEA concentrations to avoid inhibition (3). Figure 7A shows a linear double reciprocal plot of activity vs. increasing PAPS concentrations obtained using $2 \mu \mathrm{M}$ DHEA and pure SULT2A1. However, a similar plot with $20 \mu \mathrm{M}$ DHEA shows that substrate activation is observed with increasing PAPS concentrations. By contrast, Figure 7B shows that substrate activation was not observed with MBP-SULT2A1 and increasing PAPS concentrations using either $2 \mu \mathrm{M}$ or $20 \mu \mathrm{M}$ DHEA. This observation suggests that the observed substrate activation, as well as substrate inhibition, is a function of the dimerization of SULT2A1. 


\section{Discussion}

Most of the cytosolic SULTs have been reported as homodimers following purification or expression $(10,13,17)$. Structural analysis and computer modeling have indicated that the highly conserved KXXXTVXXXE (KTVE) domain is utilized as the dimerization interface in the cytosolic SULT family $(13,17)$. Generation of the MBP-SULT2A1 fusion protein inhibits dimerization without significantly altering the $K_{\mathrm{m}}$ or $V_{\max }$ of the enzyme for DHEA sulfation. However, in contrast to the pure dimeric enzyme, substrate inhibition was not observed with increasing DHEA concentrations utilizing the MBP-SULT2A1 fusion protein (Figure 4). To further address the role of dimerization in substrate inhibition, the sulfation of E2 sulfation by mouse SULT1E1 was examined. Mouse SULT1E1 is the only reported naturally occurring monomeric SULT owing to mutations in the KTVE domain (17). Whereas both the mouse and human SULT1E1 isoforms have $K_{\mathrm{m}}$ values for E2 sulfation of approximately $5 \mathrm{nM}(10,21,22)$, mouse SULT1E1 did not demonstrate substrate inhibition during E2 sulfation at concentrations in excess of concentrations where substrate inhibition is observed with the dimeric human SULT1E1 isoform (10). Although substrate inhibition is not observed with all the various SULT-substrate combinations (9), homodimer formation is apparently involved with allowing substrate inhibition with increasing DHEA concentrations in SULT2A1 and also in E2 sulfation by SULT1E1 (10).

SULT2A1 exists in at least two separate conformations, an 'open' conformation occurring in the absence of bound PAPS or PAP and a 'closed' conformation that occurs following a structural rearrangement elicited by PAPS binding (9). Movement of the loop3 peptide that forms the outer surface of the active site is primarily responsible for the decreased volume of the binding pockets. The volumes of both the PAPS and substrate binding pockets are decreased approximately $40 \%$ in the closed conformation as compared with the open. Molecular modeling indicates that two DHEA molecules can bind in the open conformation of the substrate binding pocket as compared with one in the closed conformation $(9,12)$. The stoichiometry studies in this report support the modeling studies. Using both the dimeric SULT2A1 and MBP-SULT2A1, two molecules of DHEA bound in the absence of PAP. Both the dimeric SULT2A1 and the MBP-SULT2A1 fusion protein bound only one DHEA molecule if they were previously incubated with molar excess amounts of PAP consistent with the decreased volume of the binding site in the closed conformation. A small drop in fluorescence was observed with the initial addition of DHEA to the SULT2A1 dimer that was not observed with the MBP-SULT2A1 monomer. This might result from DHEA binding at a non-specific hydrophobic site formed at the dimer interface because the stoichiometry is approximately 0.3-0.5 DHEA molecules bound per dimer.

Dimeric SULT2A1 also generated two $K_{\mathrm{d}}$ values for DHEA binding in the open conformation, whereas the MBP-SULT2A1 only produced a single $K_{\mathrm{d}}$ value. With PAP bound both enzymes showed only a single $K_{\mathrm{d}}$ for DHEA binding. The data suggest that although two DHEA molecules can bind in the open substrate binding pocket of SULT2A1, in the homodimer binding of the second DHEA molecule alters the reaction possibly through inhibiting access of DHEA to the catalytic site. In the MBP-SULT2A1 fusion protein, the second DHEA molecule binds either with a significantly lower affinity to the same site as in native SULT2A1 so that a $K_{\mathrm{d}}$ was not be measurable or it binds in a different position and does not elicit a change in fluorescence or have an effect on DHEA binding at the catalytic site.

Because most DHEA sulfation assays are conducted in the presence of high PAPS concentrations ( $\left.>20 \times K_{\mathrm{m}}\right)$, the formation of the closed conformation of the enzyme is favored (9). At low DHEA concentrations, binding of DHEA to the higher affinity catalytic site results in increased DHEA-sulfate formation. As the DHEA concentration increases in 
the reaction, binding at the second lower affinity allosteric DHEA site becomes significant and decreases the reaction rate, possibly by inhibiting access of DHEA to the active site. However, at high DHEA concentrations the rate of DHEA binding to the low affinity allosteric site will be influenced by PAPS binding that results in the closed conformation of the active site and apparently prevents DHEA binding to the allosteric site. Therefore, the low constant reaction rate observed at high DHEA levels results from an interaction of the steady state rates of PAPS binding and DHEA binding to the allosteric site. We also expect that the enzyme with two DHEA molecules bound will be able to bind PAPS and generate DHEA-sulfate because raloxifene, which can only bind productively in the open conformation of SULT2A1, is readily sulfated (9).

Interaction of the two DHEA binding sites with PAPS can also explain the substrate activation observed with dimeric SULT2A1 at relatively high DHEA concentrations and increasing PAPS concentrations. DHEA sulfation has generally been assayed at a concentration of approximately $3 \mu \mathrm{M}$ to avoid substrate inhibition $(2,3)$, a concentration that would favor optimal binding at the catalytic site as compared with the allosteric site, especially at high PAPS concentrations that favor formation of the closed conformation of the enzyme. As shown in Figure 5, at the relatively low concentration of $2 \mu \mathrm{M}$ DHEA increasing PAPS concentrations do not generate substrate activation because there would be little DHEA binding of the allosteric site. However, at $20 \mu \mathrm{M}$ DHEA both DHEA binding sites should be highly bound. DHEA sulfation increases with PAPS concentration until approximately $0.6 \mu \mathrm{M}$ when the rate increases. This is consistent with increased PAPS binding causing an increase in the closed conformation of the active site and decreased binding of DHEA to the allosteric site.

The lack of substrate inhibition and substrate activation in the MBP-SULT2A1 monomer is apparently associated with the loss of subunit interactions at the KTVE dimerization interface. The KTVE interface is contiguous with the motile loop3 (Figure 2). Loop3 is the most flexible region in SULT2A1 (Figure 3) and the KTVE region also shares some of that flexibility. The lack of subunit interaction can explain the loss of substrate activation and inhibition if DHEA binding in the allosteric site is altered sufficiently by the lack of dimerization to decrease DHEA binding at the allosteric site. Because the $K_{\mathrm{m}}$ and $V_{\max }$ for DHEA sulfation are not affected, the catalytic mechanism does not appear to be significantly affected, suggesting that only the allosteric site is involved.

Our laboratory has previously reported (9) that two DHEA molecules can be modeled into the open conformation of the SULT2A1 active site, one in the catalytic site and one in the apparent allosteric site. However, all reported crystal structures of SULT2A1 are resolved without dimer formation utilizing the KTVE site $(12,18,19,23)$. Therefore, any structural changes elicited by formation of the interface at the KTVE domain are probably not represented in the resolved structures. The reported structures most likely represent structures, at least of the substrate binding pocket, similar to that of MBP-SULT2A1. The stoichiometry binding studies indicate that two DHEA molecules can bind to MBPSULT2A1; however, binding of the non-catalytic DHEA is probably not in an orientation or with an affinity to affect catalytic activity.

Several possible mechanisms for substrate inhibition have been suggested including deadend ternary complexes (10), allosteric substrate binding (24) and alternative substrate orientations (12). Our data suggest that at least for SULT2A1, the formation of the homodimer has an important role. This could relate to the size of the binding pockets and the changes in the pocket associated with both dimerization and PAPS binding. The mechanism of substrate inhibition might be different in different isoforms, because the substrate interactions are probably substrate and isoform selective. Careful analysis of substrate 
inhibition with multiple substrates as well as in different SULT isoforms is needed to better understand its role in the mechanism of sulfonate conjugation.

\section{References}

1. Comer KA, Falany JL, Falany CN. Cloning and expression of human liver dehydroepiandrosterone sulphotransferase. Biochem J. 1993; 289:233-40. [PubMed: 7678732]

2. Falany CN, Vazquez ME, Kalb JM. Purification and characterization of human liver dehydroepiandrosterone sulphotransferase. Biochem J. 1989; 260:641-6. [PubMed: 2764897]

3. Falany CN, Wheeler J, Oh TS, Falany JL. Steroid sulfation by expressed human cytosolic sulfotransferases. J Steroid Biochem Mol Biol. 1994; 48:369-75. [PubMed: 8142314]

4. Comer KA, Falany CN. Immunological characterization of dehydroepiandrosterone sulfotransferase from human liver and adrenal. Mol Pharmacol. 1992; 41:645-51. [PubMed: 1569919]

5. Falany C, Comer K, Dooley T, Glatt H. Human dehydroepiandrosterone sulfotransferase: purification, molecular cloning, and characterization. Ann NY Acad Sci. 1995; 774:59-72. [PubMed: 8597484]

6. Falany CN. Enzymology of human cytosolic sulfotransferases. FASEB J. 1997; 11:206-16. [PubMed: 9068609]

7. Fang HL, Strom SC, Ellis E, Duanmu Z, Fu J, Duniec-Dmuchowski Z, Falany CN, Falany JL, Kocarek TA, Runge-Morris M. Positive and negative regulation of human hepatic hydroxysteroid sulfotransferase (SULT2A1) gene transcription by rifampicin: roles of hepatocyte nuclear factor 4alpha and pregnane X receptor. J Pharmacol Exp Ther. 2007; 323:586-98. [PubMed: 17687072]

8. Fang HL, Strom SC, Cai H, Falany CN, Kocarek TA, Runge-Morris M. Regulation of human hepatic hydroxysteroid sulfotransferase gene expression by the peroxisome proliferator-activated receptor alpha transcription factor. Mol Pharmacol. 2005; 67:1257-67. [PubMed: 15635043]

9. Cook I, Leyh T, Kadlubar S, Falany C. Structural rearrangement of SULT2A1: effects on dehydroepiandrosterone and raloxifene sulfation. Horm Mol Biol Clin Invest. 2010; 1:81-7.

10. Zhang H, Varlamova O, Vargas FM, Falany CN, Leyh TS, Varmalova O. Sulfuryl transfer: the catalytic mechanism of human estrogen sulfotransferase. J Biol Chem. 1998; 273:10888-92. [PubMed: 9556564]

11. Barnett AC, Tsvetanov S, Gamage N, Martin JL, Duggleby RG, McManus ME. Active site mutations and substrate inhibition in human sulfotransferase 1A1 and 1A3. J Biol Chem. 2004; 279:18799-805. [PubMed: 14871892]

12. Lu LY, Hsieh YC, Liu MY, Lin YH, Chen CJ, Yang YS. Identification and characterization of two amino acids critical for the substrate inhibition of human dehydroepiandrosterone sulfotransferase (SULT2A1). Mol Pharmacol. 2008; 73:660-8. [PubMed: 18042734]

13. Weitzner B, Meehan T, Xu Q, Dunbrack RL Jr. An unusually small dimer interface is observed in all available crystal structures of cytosolic sulfotransferases. Proteins. 2009; 75:289-95. [PubMed: 19173308]

14. Allali-Hassani A, Pan PW, Dombrovski L, Najmanovich R, Tempel W, Dong A, Loppnau P, Martin F, Thornton J, Edwards AM, Bochkarev A, Plotnikov AN, Vedadi M, Arrowsmith CH. Structural and chemical profiling of the human cytosolic sulfotransferases. PLoS Biol. 2007; 5:e97. [PubMed: 17425406]

15. Cook IT, Duniec-Dmuchowski Z, Kocarek TA, Runge-Morris M, Falany CN. 24Hydroxycholesterol sulfation by human cytosolic sulfotransferases: formation of monosulfates and disulfates, molecular modeling, sulfatase sensitivity, and inhibition of liver $\mathrm{x}$ receptor activation. Drug Metab Dispos. 2009; 37:2069-78. [PubMed: 19589875]

16. Bell, J.; Bell, E., editors. Proteins and enzymes. Englewood Cliffs, NJ: Prentice-Hall Inc; 1988.

17. Petrotchenko EV, Pedersen LC, Borchers CH, Tomer KB, Negishi M. The dimerization motif of cytosolic sulfotransferases. FEBS Lett. 2001; 490:39-43. [PubMed: 11172807]

18. Pedersen LC, Petrotchenko EV, Negishi M. Crystal structure of SULT2A3, human hydroxysteroid sulfotransferase. FEBS Lett. 2000; 475:61-4. [PubMed: 10854859]

19. Rehse PH, Zhou M, Lin SX. Crystal structure of human dehydroepiandrosterone sulphotransferase in complex with substrate. Biochem J. 2002; 364:165-71. [PubMed: 11988089] 
20. Falany JL, Greer H, Kovacs T, Sorscher EJ, Falany CN. Elevation of hepatic sulphotransferase activities in mice with resistance to cystic fibrosis. Biochem J. 2002; 364:115-20. [PubMed: 11988083]

21. Falany CN, Krasnykh V, Falany JL. Bacterial expression and characterization of a cDNA for human liver estrogen sulfotransferase. J Steroid Biochem Mol Biol. 1995; 52:529-39. [PubMed: 7779757]

22. Kakuta Y, Pedersen LC, Chae K, Song WC, Leblanc D, London R, Carter CW, Negishi M. Mouse steroid sulfotransferases: substrate specificity and preliminary X-ray crystallographic analysis. Biochem Pharmacol. 1998; 55:313-7. [PubMed: 9484797]

23. Chang HJ, Shi R, Rehse P, Lin SX. Identifying androsterone (ADT) as a cognate substrate for human dehydroepiandrosterone sulfotransferase (DHEA-ST) important for steroid homeostasis: structure of the enzyme-ADT complex. J Biol Chem. 2004; 279:2689-96. [PubMed: 14573603]

24. Gamage NU, Duggleby RG, Barnett AC, Tresillian M, Latham CF, Liyou NE, McManus ME, Martin JL. Structure of a human carcinogen-converting enzyme, SULT1A1. Structural and kinetic implications of substrate inhibition. J Biol Chem. 2003; 278:7655-62. [PubMed: 12471039] 

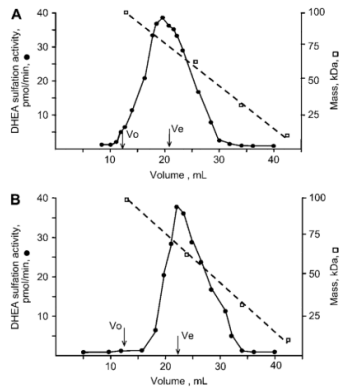

Figure 1.

Size exclusion chromatography of SULT2A1 and MBP-SULT2A1. A Sephadex G-100 size exclusion column $(40 \mathrm{~mL})$ was equilibrated with $10 \mathrm{mM}$ potassium phosphate $(\mathrm{pH} 7.4), 50$ $\mathrm{mM} \mathrm{NaCl}$, and $10 \%$ glycerol. The column was calibrated with Bio-Rad molecular weight standards (Dextran blue, bovine serum albumin, carbonic anhydrase, cytochrome C). MBPSULT2A1 fusion protein (A) or native SULT2A1 (B) was loaded on the column in a volume of $0.2 \mathrm{~mL}$. The column was eluted with the same buffer and the elution of enzyme was monitored by DHEA sulfation activity. The monomeric MBP-2A1 fusion protein eluted at $20.9 \pm 0.9 \mathrm{~mL}$ and the dimeric SULT2A1 eluted at 22.0 $\pm 1.1 \mathrm{~mL}(\mathrm{n}=3)$. 


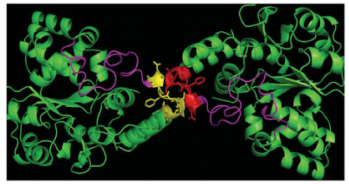

Figure 2.

Model of SULT2A1 homodimer. A model of the SULT2A1 homodimer was generated utilizing MOE. The dimer was modeled by orienting the resolved monomer structures of SULT2A1 (1J99) with the interface at the KTVE site based on the dimer interface in SULT1E1 (1G3M). Green represents the core of the enzyme, the red and yellow peptides show the dimerization domains of each subunit and the loop3 peptide is shown in purple. 


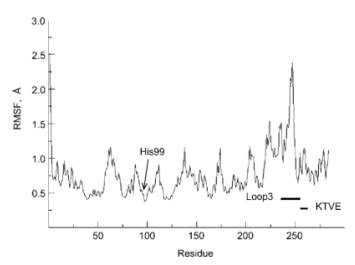

Figure 3.

Molecular dynamic modeling of SULT2A1. Dynamic simulation of SULT2A1 was generated using the Amber energy field in MOE and a time-frame of $5 \mathrm{~ns}$. The root square mean fluxuation (RMSF) was calculated for the carbon backbone atoms. Most of the enzyme demonstrated less than a $0.1 \mathrm{~nm}$ RMSF over the entire simulation. The loop3 region demonstrated a greater degree of dynamics with a RMSF value almost twice the average for the rest of the enzyme. A minor spike in dynamics was also observed in the KTVE region adjacent to loop3. 


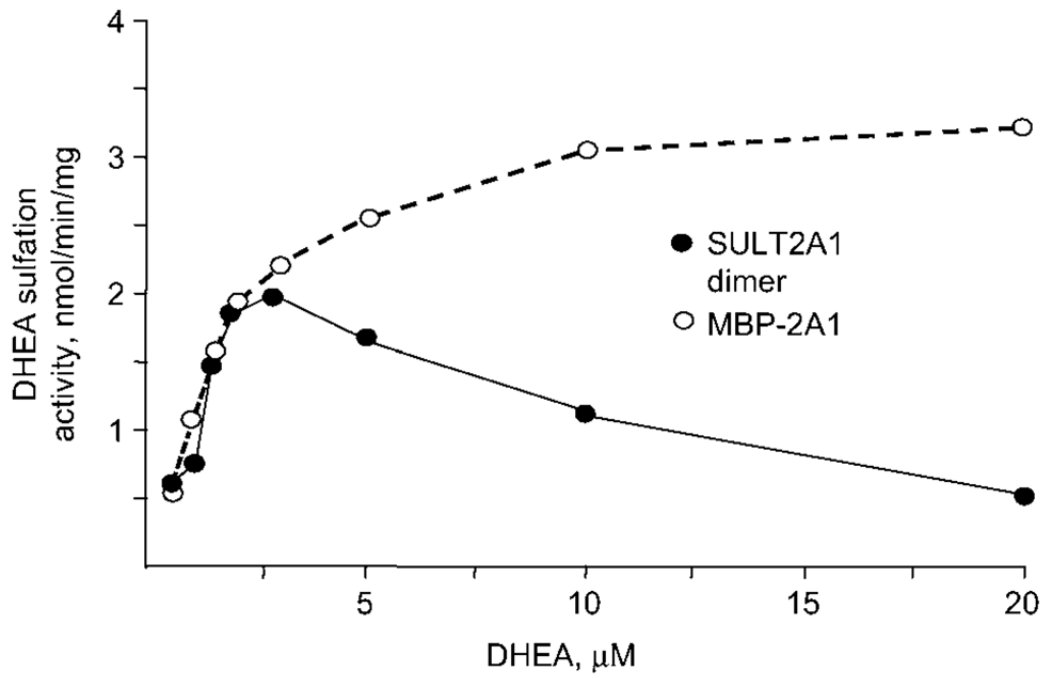

Figure 4.

Sulfation of increasing DHEA concentrations by SULT-2A1 and MBP-SULT2A1. DHEA sulfation activity of SULT-2A1 (closed circles) and MBP-SULT2A1 (open circles) was assayed in $10 \mathrm{mM}$ potassium phosphate $(\mathrm{pH} 7.2)$ and $5 \mathrm{mM} \mathrm{MgCl}_{2}$, with $10 \mu \mathrm{M}$ PAPS and DHEA $(0.1-20 \mu \mathrm{M})$. Reactions were started with the addition of enzyme $(0.5 \mu \mathrm{g})$ and incubated at $37^{\circ} \mathrm{C}$ for $10 \mathrm{~min}$. Each point represents the average of at least three sets of reactions done in duplicate. 


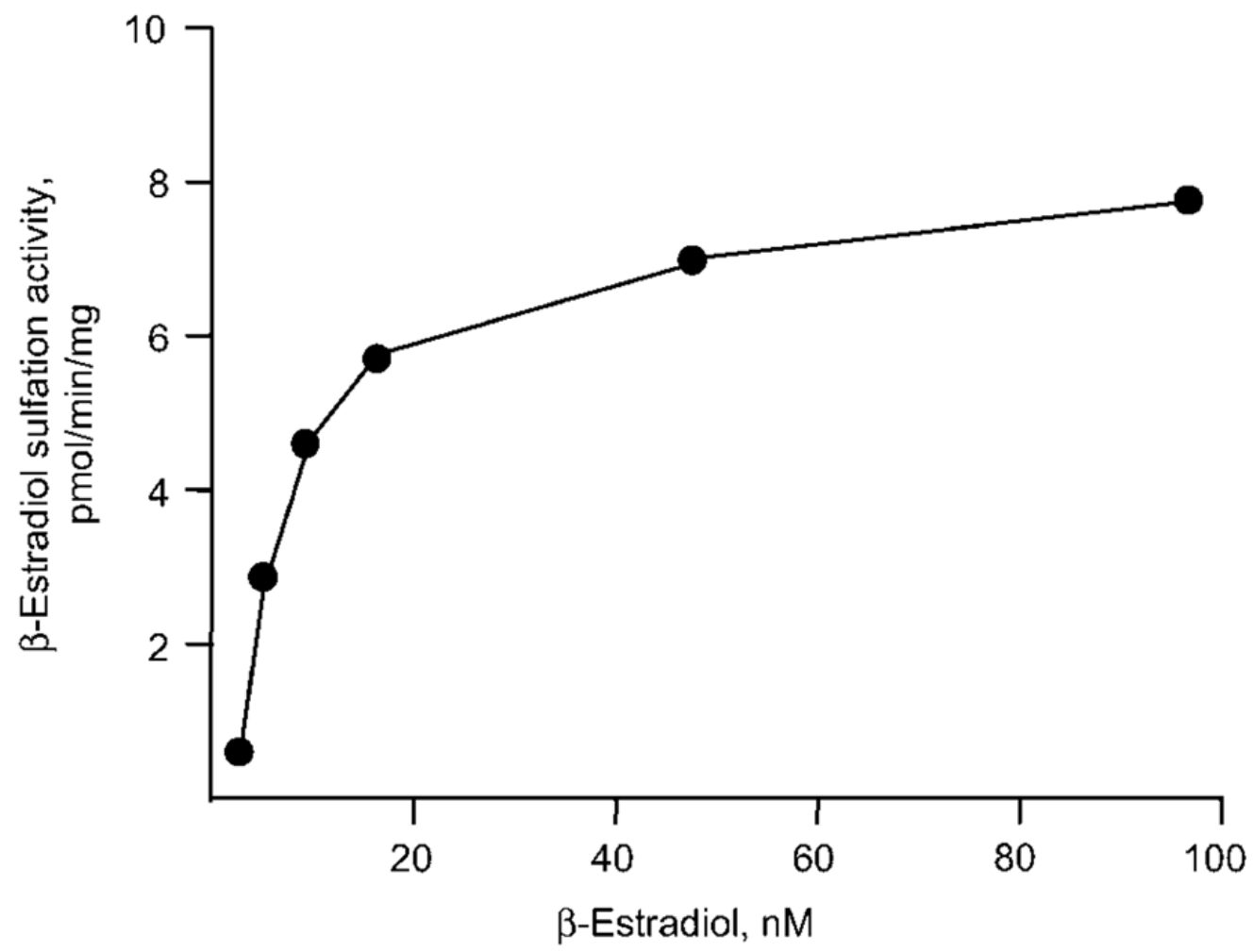

Figure 5.

$\beta$-Estradiol sulfation by mouse SULT1E1. Cytosol prepared from the liver of a cystic fibrosis $\triangle F 508$ mouse over-expressing SULT1E1 (20) was assayed for E2 sulfation activity. The reactions were implemented as described previously (21) except that E2 concentrations were varied from 2 to $100 \mathrm{nM}$. Each point is the average E2 sulfation rate using three separate mouse liver cytosols and each set of reactions performed in triplicate. 

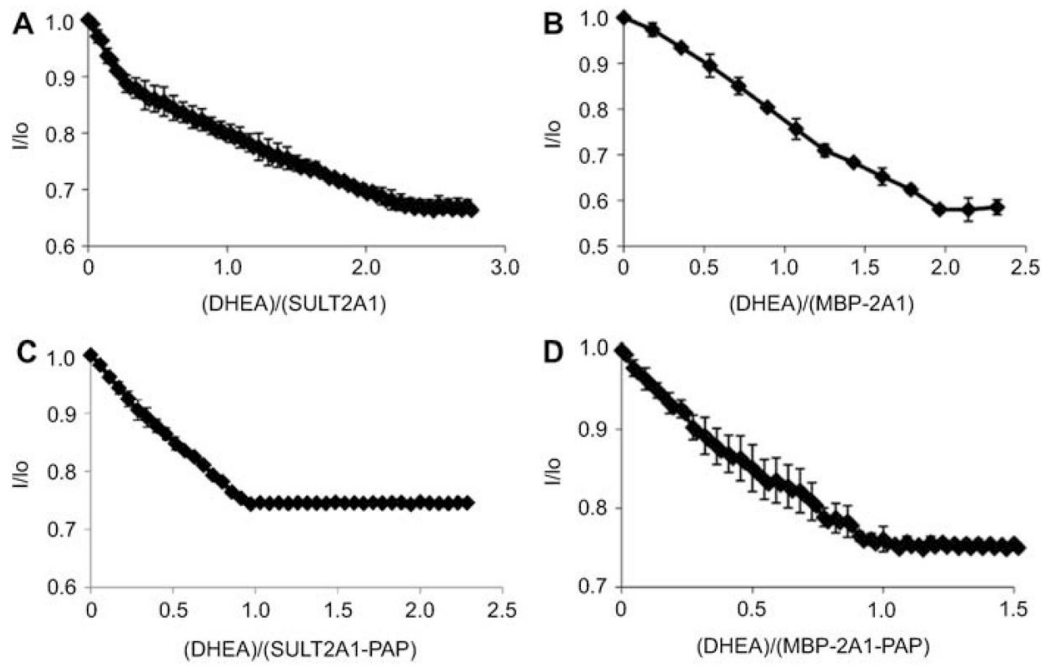

Figure 6.

Determination of stoichiometry of substrate binding with SULT2A1 and MBP-SULT2A1. The stoichiometry of DHEA and PAP/ PAPS binding to SULT2A1 were estimated using changes in intrinsic fluorescence of the pure proteins. The pure MBP-SULT2A1 and SULT2A1 were generated as described in the methods section. Changes in intrinsic fluorescence were monitored with a Perkin-Elmer LS-5 fluorescence spectrophotometer with excitation at $275 \mathrm{~nm}$ and detection at $340 \mathrm{~nm}$. For stoichiometry studies, $10 \mu \mathrm{M}$ SULT2A1 monomers in $10 \mathrm{mM}$ phosphate buffer, $\mathrm{pH} 7.4$ with $150 \mathrm{mM} \mathrm{NaCl}$ and $1 \mathrm{mM}$ DTT in a volume of $3 \mathrm{~mL}$ were used. Increasing amounts of DHEA or PAP/PAPS were added in $2 \mu \mathrm{L}$ aliquots and allowed to equilibrate for $3 \mathrm{~min}$ before fluorescence was measured. To measure substrate binding to the closed conformation of SULT2A1, the enzyme was preincubated with $20 \mu \mathrm{M}$ PAP before the titration with DHEA. The effect of DHEA on the binding of PAP to SULT2A1 was also analyzed after preincubation of the enzyme with an excess of substrate (e.g., $20 \mu \mathrm{M}$ DHEA). 

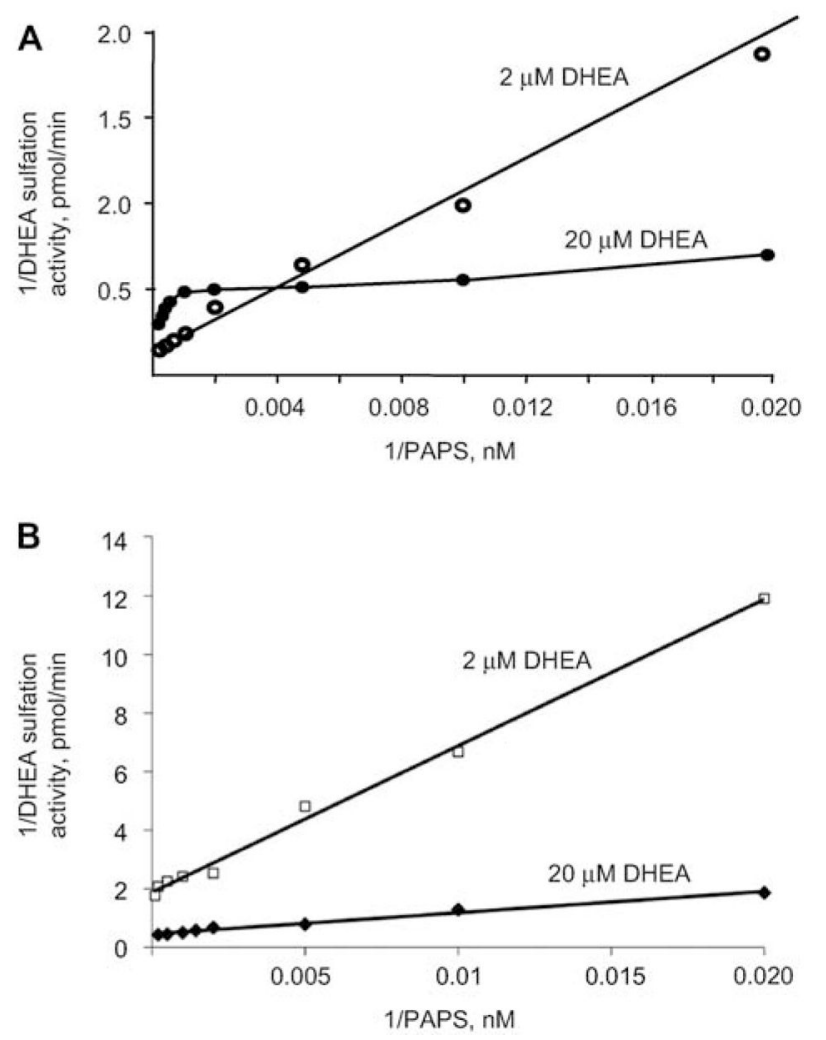

Figure 7.

Substrate activation with increasing PAPS concentration in DHEA sulfation with SULT2A1 and MBP-SULT2A1. DHEA sulfation activity was measured as described in Methods. Activity was assayed a fixed concentration of DHEA $(2 \mu \mathrm{M}$ for open circles and $20 \mu \mathrm{M}$ for closed circles) and increasing concentrations of PAPS (50 nM to $20 \mu \mathrm{M})$. Reactions were started with addition of $0.5 \mu \mathrm{g}$ of enzyme and incubated for $10 \mathrm{~min}$ at $37^{\circ} \mathrm{C}$. 


\section{Table 1}

Dissociation constants $\left(K_{\mathrm{d}}\right)$ for substrates binding to SULT2A1. $K_{\mathrm{d}}$ values were calculated for binding of DHEA, PAPS and PAP to dimeric SULT2A1, to SULT2A1 preincubated with PAP, to monomeric MBPSULT2A1 and to MBP-SULT2A1 preincubated with PAP. The $K_{\mathrm{d}}$ values were estimated from changes in intrinsic fluorescence of the substrates binding to the different forms of SULT2A1 as described in the methods section.

\begin{tabular}{lcll}
\hline Enzyme & \multicolumn{1}{l}{$\boldsymbol{K}_{\mathbf{d}}, \mathbf{n M}$} & & \\
\cline { 2 - 4 } & $\mathbf{D H E A}$ & PAPS & PAP \\
\hline SULT2A1 & $290 \pm 40$ & $250 \pm 30$ & $550 \pm 50$ \\
SULT2A1-PAP & $3100 \pm 500$ & & \\
MBP-SULT2A1 & $280 \pm 20$ & $260 \pm 40$ & $400 \pm 60$ \\
MBP-SULT2A1-PAP & $390 \pm 60$ & - & - \\
\hline
\end{tabular}

